\title{
Analysis of Single-Unit Responses to Emotional Scenes in Human Ventromedial Prefrontal Cortex
}

\author{
Hiroto Kawasaki ${ }^{1}$, Ralph Adolphs ${ }^{1,2}$, Hiroyuki Oya ${ }^{1}$, \\ Christopher Kovach ${ }^{1}$, Hanna Damasio', Olaf Kaufman', \\ and Matthew Howard, III
}

\begin{abstract}
Lesion and functional imaging studies in humans have shown that the ventral and medial prefrontal cortex is critically involved in the processing of emotional stimuli, but both of these methods have limited spatiotemporal resolution. Conversely, neurophysiological studies of emotion in nonhuman primates typically rely on stimuli that do not require elaborate cognitive processing. To begin bridging this gap, we recorded from a total of 267 neurons in the left and right orbital and anterior cingulate cortices of four patients who had chronically implanted depth electrodes for moni-
\end{abstract}

\section{INTRODUCTION}

The primate ventromedial prefrontal cortex encompasses the medial sector of the orbital cortex and the gyrus rectus on the ventral surface of the frontal lobe, as well as contiguous medial sectors immediately dorsal to the orbital cortex and extending into the subgenual cingulate cortex. The functional connectivity of this set of regions identifies it as a key locus for triggering and regulating visceral and autonomic responses related to emotions (Öngür \& Price, 2000).

In humans, this region has been shown to participate in the guidance of decision making by emotion (O'Doherty, Kringelbach, Rolls, Hornak, \& Andrews, 2001; Bechara, Damasio, Damasio, \& Anderson, 1994; Damasio, 1994) and in the recognition of emotion from voice and facial expression (Hornak, Rolls, \& Wade, 1996). The subgenual cingulate cortex is consistently activated in functional imaging studies during depression and sadness (Damasio et al., 2000; Drevets et al., 1997). There is a general indication that the medial and ventral sectors of the prefrontal cortex may be especially important for processing aversive emotions (Kawasaki et al., 2001; Marinkovic, Trebon, Chauvel, \& Halgren, 2000): Facial expressions of fear (Vuilleumier, Armony, Driver, \& Dolan, 2001) and anger (Blair, Morris, Frith,

\footnotetext{
${ }^{1}$ University of Iowa College of Medicine, ${ }^{2}$ California Institute of Technology
}

toring epilepsy. Peristimulus activity was recorded to standardized, complex visual scenes depicting neutral, pleasant, or aversive content. Recording locations were verified with postoperative magnetic resonance imaging. Using a conservative, multistep statistical evaluation, we found significant responses in 56 neurons; 16 of these were selective for only one emotion class, most often aversive. The findings suggest sparse and widely distributed processing of emotional value in the prefrontal cortex, with a predominance of responses to aversive stimuli.
Perrett, \& Dolan, 1999) activate the right orbital cortex in functional imaging studies, and recognition of facial expressions of anger is disrupted by transcranial magnetic stimulation applied to medial prefrontal regions (Harmer, Thilo, Rothwell, \& Goodwin, 2001). Some studies pointed to differential processing of positive or negative emotions by the left or the right frontal lobes, respectively (Royet et al., 2000; Canli, Zhao, Desmond, Glover, \& Gabriel, 1999; Davidson \& Irwin, 1999; Borod et al., 1998; Davidson, 1992), or by lateral versus medial sectors of the prefrontal cortex (Northoff et al., 2000), raising the possibility that different emotions may be processed by neuroanatomically distinct regions.

Investigations of the ventral prefrontal cortex in humans pose special challenges. Lesion studies and positron emission tomography provide coarse spatiotemporal resolution, whereas functional magnetic resonance imaging (fMRI) suffers from a severe paramagnetic susceptibility artifact due to the proximity of an air-tissue interface. For this reason, most information regarding the functional role of specific neural structures in mediating emotional behavior has come from studies in laboratory animals (see Cavada \& Schultz, 2000, for reviews). In monkeys, neurons within the orbital cortex have been shown to respond selectively to a variety of sensory stimuli based on their emotional significance, independently of their basic perceptual properties (Roesch \& Olson, 2004; Wallis 
\& Miller, 2003; Cavada \& Schultz, 2000; Hikosaka \& Watanabe, 2000; Rolls, 1999; Rolls, Critchley, Mason, \& Wakeman, 1996; Thorpe, Rolls, \& Maddison, 1983), but no clear topography of such emotional responses has been found.

The above findings motivated the following questions. First, do neurons in the human prefrontal cortex respond to the emotional category of a sensory stimulus and, if so, is there selectivity to stimuli that are aversive, pleasant, or neutral? Second, if such neurons exist, is their distribution topographic? Third, might there be neuronal representations along more continuous stimulus dimensions, such as arousal and valence? Here we address these questions with electrophysiological studies of neurons recorded within the ventromedial prefrontal cortex of four rare human subjects undergoing evaluation for the treatment of intractable epilepsy. In this study, we consider "emotion category" as a property of the stimuli, rather than of the judgments that subjects make about them. Thus, for example, we would put into the "aversive" category all the stimuli to which normal subjects agree they are aversive. This approach analyzes the responses in the prefrontal cortex that we recorded as essentially stimulus evoked and sensory in nature.

\section{RESULTS}

\section{Neuroanatomy}

Detailed analyses of recording site locations were obtained by coregistration of pre- and postimplantation MR scans and reconstruction of electrode positions (Figure 1). Recording sites were located in four subregions of the ventromedial prefrontal cortex: right subgenual cingulate cortex (sgCC: Brodmann's area 24/25), right and left medial orbitofrontal cortices (mOFC: Brodmann's area 11, between the olfactory sulcus and the medial orbital sulcus), and left gyrus rectus (GR: Brodmann's area 11, medial to the olfactory sulcus).

Patient 57 had four recording sites, two in the right OFC and two in the right sgCC (Figure 1A). Patient 66 had two recording sites in the left mOFC. Patient 70 had two recording site in the left GR, and Patient 104 had four recording sites, two in the left GR and two in the right $\mathrm{mOFC}$. Recording locations at which significant responses were obtained are summarized in Figure 2.

\section{Physiology}

We used as stimuli complex, standardized scenes (Lang, Oehman, \& Vaitl, 1988) because these offer the most ecological validity with static stimuli and because they have been established to reliably signal strong and specific emotions in prior studies. Analyses of neuronal responses were carried out for the same set of stimuli in each subject (see Methods) because we wanted to

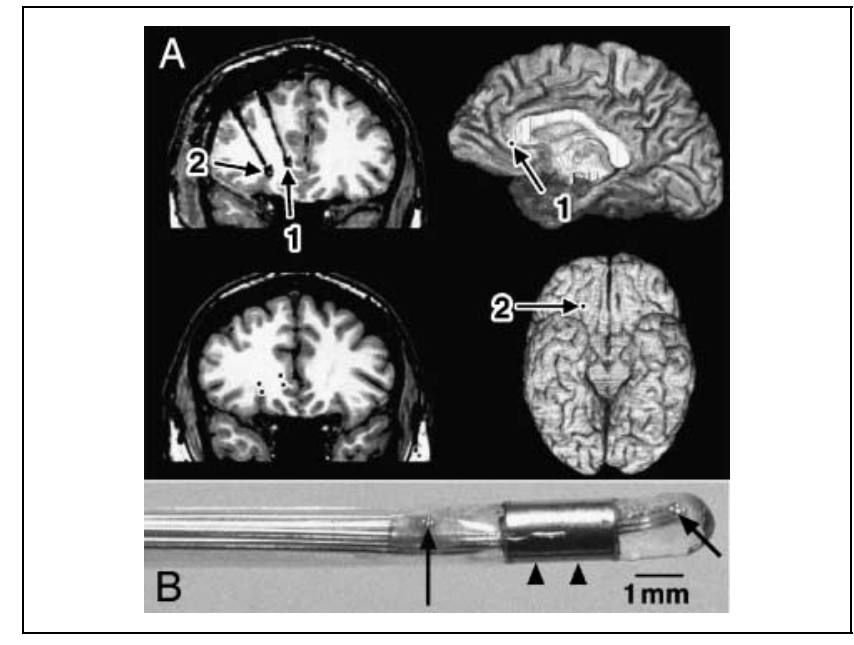

Figure 1. Neuroanatomical localization of recording sites and electrode design. (A) Individual images and three-dimensional reconstructions of the brain of Patient 57 indicating the locations at which we recorded (arrows). Identical analyses were carried out for each of the four patients, with very similar quality of images. Postimplantation scans (top left) show the actual electrodes in situ (black signal artifact on coronal scan); preimplantation scans (bottom left) indicate the clusters of recording sites (small black squares) on the intact brain, mapped from the information available in the postimplantation scans. The location of the recording sites was visualized also on three-dimensional reconstructions of the subject's brain, as shown in the examples on the right. (B) Photomicrograph of the recording electrode; the two high-impedance research-recording sites are just visible as tiny silver clusters (arrows) flanking the large platinum clinical low-impedance contact (arrowheads).

investigate responses driven by the stimuli rather than by individual differences in the cognitive judgments that subjects might make about them. Our approach thus parallels that taken for the majority of functional imaging studies of emotional stimuli and allows comparisons across the subjects from whom we recorded.

At each recording site, we were able to isolate a total of 30-50 neurons from up to four closely clustered recording channels, for a grand total of 267 neurons in the four patients: 81 in the left GR, 41 in the left mOFC, 99 in the right $\mathrm{mOFC}$, and 46 in the right sgCC. The heterogeneity of the individual stimuli used within each class of emotion (see Methods for details) ensured that the responses we found could not be attributed to a single simple visual property of the stimuli. We also confirmed quantitatively that the stimuli within different emotion categories did not differ significantly on mean luminance or color composition, and further performed a median-split analysis of neuronal responses to that half of the neutral stimuli with the lowest luminance, contrasted with that half of the neutral stimuli with the highest luminance. None of these contrasts were significant for any of the neurons responsive to emotion.

We obtained 56 (21.0\%) neurons with statistically significant responses to our visual stimuli. Of these, 
Figure 2. Overview of responses. (A) Summary of recording sites at which significant selective responses to emotional stimuli were found. The upper middle panel shows a projection of the recording site of the right subgenual cingulate cortex (R sgCC) onto the sagittal surface, and the left upper panel indicates the number of neurons with particular response selectivities found in this location. The left lower, the right upper, and the right lower panels show bar graphs of the number of selective neurons found in the right medial orbitofrontal cortex ( $\mathrm{R} \mathrm{mOFC}$ ), the left gyrus rectus (L GR), and the left medial orbitofrontal cortex (L mOFC), respectively. Projections of the recording sites onto the ventral surface of the brain are shown in the lower middle panel. White bars represent neurons that increased their firing rates in response to visual stimuli, and the black bar represents a neuron whose response showed a decrease in firing rate. Each bar graph also indicates the total number of neurons that are isolated for each recording site. (B) Total number of neurons with selective responses to the three different emotion categories across all recording sites, broken down by left and right hemisphere. Although the majority of neurons responded to aversive stimuli, their distribution did not differ between hemispheres. (C) Percentages of neuronal response classes across all recording sites. $\mathrm{R}$-visually responsive but not emotion selective; $\mathrm{A}=$ selective to aversive stimuli; $\mathrm{P}=$ selective to pleasant stimuli; $\mathrm{N}=$ selective to neutral stimuli.
16 neurons (6.0\%) responded selectively to one of the emotion categories: 9 (3.4\%) to aversive stimuli, 1 $(0.4 \%)$ to pleasant stimuli, and $6(2.2 \%)$ to neutral stimuli (Figures 2 and 3). Of these, 8, 1 and 6 neurons increased spike rates selectively to aversive, pleasant, and neutral stimuli, respectively, and 1 neuron decreased neural activity selectively to aversive stimuli. The numbers of selective neurons were not distributed equally among emotion categories $\left(\chi^{2}=16.9\right.$, $d f=2, p<.0003)$. There was no significant difference in the distribution of emotion-category-selective neurons between the two hemispheres (generalized Fisher's exact statistic from re-randomization, $p=.35$ ) (Figure 2B).

We quantified the strength of emotion effects on spike firing by assessing how well an ideal observer could decide from the spike rates whether a stimulus belonged to an emotion category. This measure was obtained from receiver operating characteristic (ROC) analyses of the selective neurons (see Methods for details). We computed the lowest possible probability of error $\left(P_{\text {error }}\right)$, using the optimal performance of the ideal observer model. The value of $P_{\text {error }}$ can range from 0 to .5 , with $P_{\text {error }}=.5$ indicating chance performance and $P_{\text {error }}=0$ indicating perfect discrimination. $P_{\text {error }}$ of selective OFC neurons ranged from .23 to .43 $(.34 \pm .06$, mean $\pm S D$, Figure $3 \mathrm{E}$ and $\mathrm{F})$. The means of $P_{\text {error }}$ for responses to the three emotion categories did not differ significantly from each other (one-way ANOVA, $p>.05)$.

Response latencies varied widely: latencies for responses to aversive pictures ranged from 320 to $890 \mathrm{msec}$ (mean $563 \pm 207 \mathrm{msec}, n=9$ ), that of a response to pleasant was $360 \mathrm{msec}$ and those of responses to neutral ranged from 30 to $850 \mathrm{msec}$ (mean $443 \pm 387$ msec, $n=6$ ). Across all responses, there was no significant effect of emotion category on response latency (one-way ANOVA, $p>.05$ ). Figure 4 plots the histogram of response latency in the poststimulus epoch.

In one subject (Subject 104), we analyzed the correlation between neural activity and valence or arousal scores of each stimulus (Figure 5). This addressed the further question as to whether responses might covary 


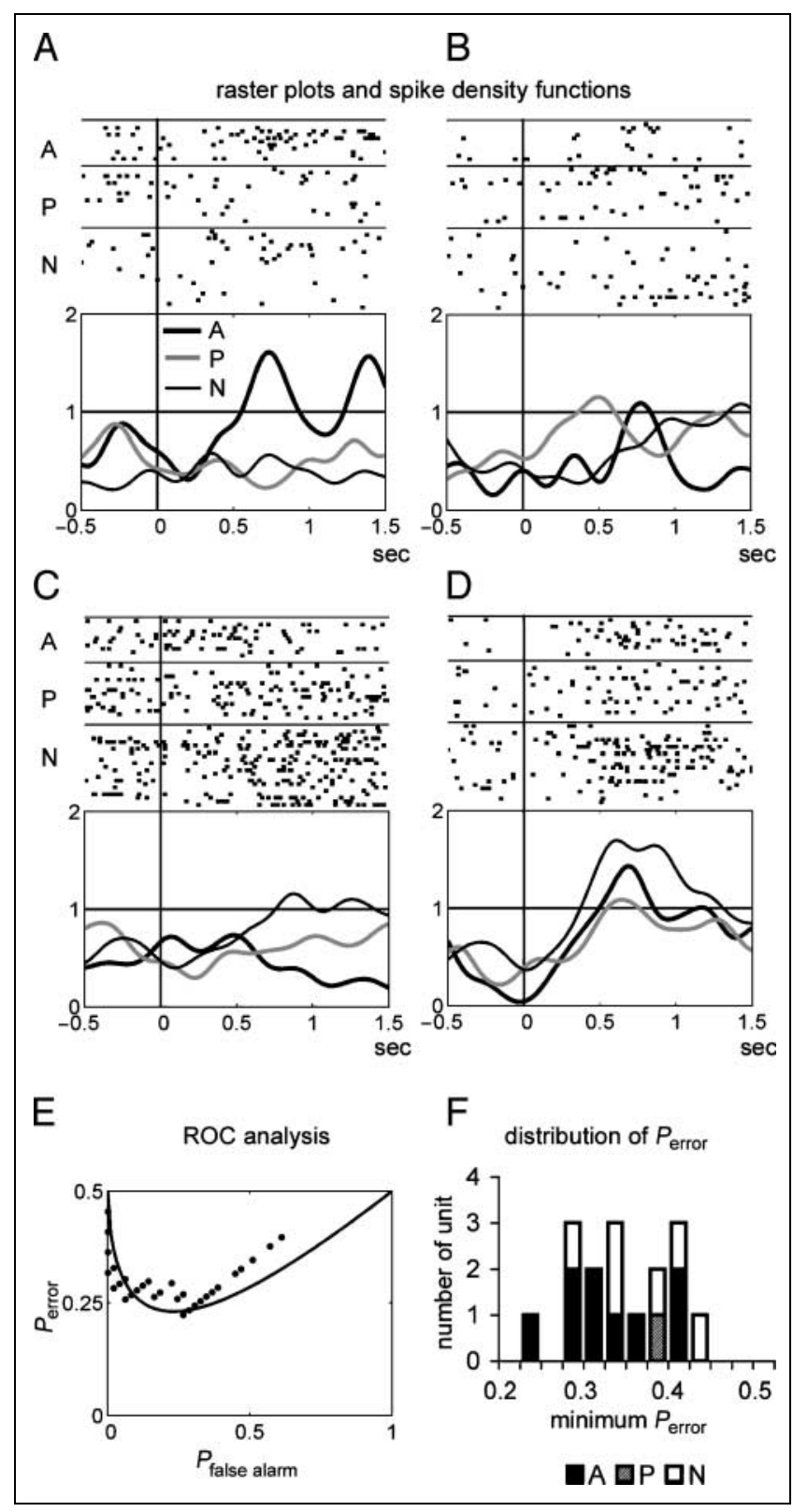

Figure 3. Single-unit responses to emotional stimuli. These panels show individual examples of rasters and spike density functions. The rasters (top) are subdivided into responses to aversive (A), pleasant $(\mathrm{P})$, or neutral $(\mathrm{N})$ stimuli, as are the instantaneous spike rates below them (thick black line, aversive; thick gray line, pleasant; thin black line, neutral). The $x$ axis shows time relative to stimulus onset. The $y$ axis gives normalized spike rate; 1 represents the $p=.05$ level estimated from the distribution of the prestimulus baseline spike rates. Example unit responses are shown for a unit (A) selective to aversive stimuli from 550 to $990 \mathrm{msec}$ after onset of stimuli, (B) selective to pleasant stimuli from 360 to $600 \mathrm{msec}$ (the brief subsequent response to aversive stimuli did not reach our selection criteria for selective response), (C) selective to neutral stimuli from 760 to $1020 \mathrm{msec}$, (D) responsive but nonselective. (E) An example of our ROC analysis: $P_{\text {error }}$ was plotted against $P_{\text {false alarm }}$ for the unit shown in (A). A curve was fitted assuming an equal variance model. The ideal observer could achieve a minimum $P_{\text {error }}=.23$. (F) The bar graph shows the distribution of $P_{\text {error }}$ of each emotion category. Means of $P_{\text {error }}$ of three emotion categories did not differ significantly from each other (one-way ANOVA, $p=.34$ ).

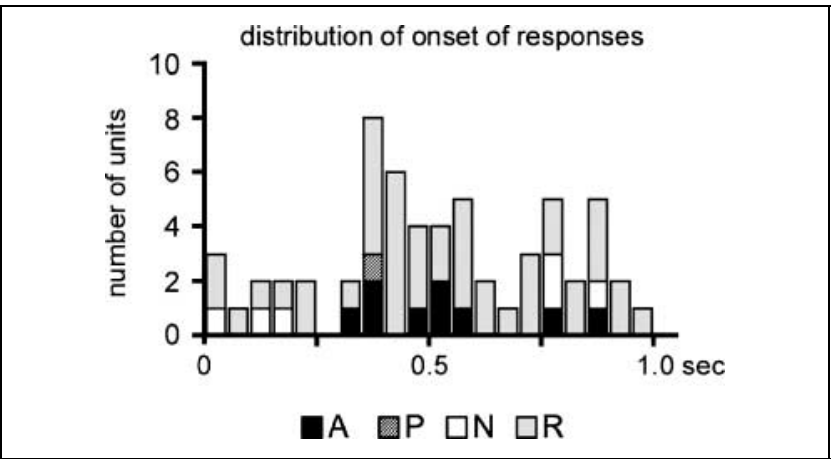

Figure 4. Histogram of response latencies. There was not a significant effect of emotion category on response latency (one-way ANOVA, $p>.05$ ). A (black) $=$ selective to aversive stimuli; $\mathrm{P}($ dark gray $)=$ selective to pleasant stimuli; $\mathrm{N}$ (white) $=$ selective to neutral stimuli; R (light gray)-nonspecific visual response.

according to the dimensional rating of the stimuli, rather than the emotional categories we used. In this subject, there were 21 responses in 18 units including 4 selective responses ( 2 to aversive and 2 to neutral). None of these responses had significant correlation to either
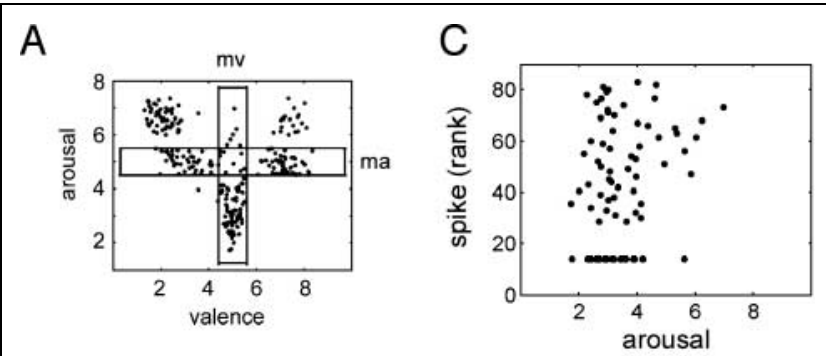

B
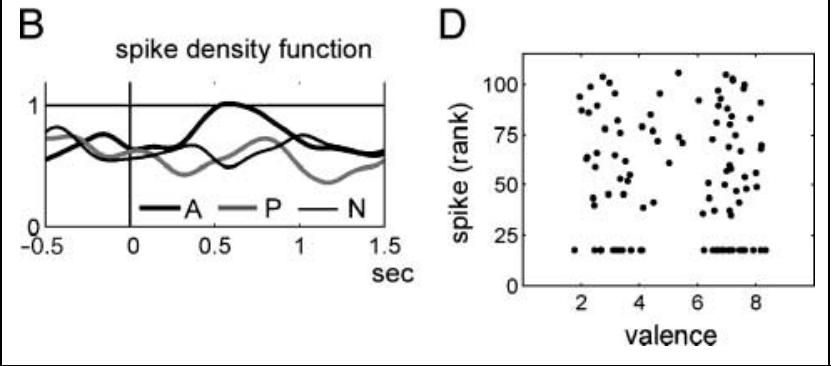

Figure 5. Response analysis by valence and arousal in Subject 104. (A) Distribution of all stimuli in a valence-arousal space. Rectangular regions indicate subsets of stimuli used in the correlational analyses shown below. Correlations with valence were computed on the moderate arousal group ("ma," $4.5<$ arousal $<5.5, n=106$ ) (horizontal rectangle). Correlations with arousal were computed on the moderate valence group ("mv," $4.5<$ valence $<5.5, n=83$ ) (vertical rectangle). (B) A sample unit in which we conducted correlational analyses. (C) Rank of spike firing of the unit shown in (B) was plotted against arousal scores of stimuli in the moderate valence group (vertical rectangle in (A) and C), and (D) against valence scores of stimuli in the moderate arousal group [horizontal rectangle in (A)]. The neuron shown in (B) selectively responded to aversive stimuli from 530 to $650 \mathrm{msec}$ and the correlation analysis was performed on the responses within same time window. 
valence or arousal ratings of stimuli (Spearman's rank correlation, $p>.05$ ).

\section{DISCUSSION}

Recording from 267 neurons in four neurosurgical patients, we were able to demonstrate statistically significant responses to visual stimuli in 56 neurons. A detailed series of MRI scans were obtained both pre- and postimplantation, allowing us to localize the recording sites with precision. We conclude that:

1. Several regions in the ventral and medial prefrontal cortex in both left and right hemisphere participate in encoding the emotional significance of complex visual stimuli.

2. Single neurons within these regions show responses to visual stimuli that are selective for aversive, pleasant, or neutral stimuli. The largest proportion of neurons showed responses selective for aversive stimuli. The selectivity we observed could not be accounted for by simpler valence or arousal dimensions from a preliminary study in one of the patients, nor was it attributable to lower-level features of the stimuli or to their mean luminance (which would cut across emotion categories, rather than yield emotion-specific responses).

3. There was no evidence for a regional neuroanatomical segregation of response selectivity. There was no evidence for clustering of response selectivity among those neurons recorded from the same location. These conclusions are of course limited by the small numbers of locations from which we recorded.

The study had two main limitations: First, we recorded from epileptic patients rather than healthy subjects; for obvious reasons it is impossible to record intracranially in humans outside this clinical setting. We ensured that the tissue from which we recorded would nonetheless have been physiologically healthy in three ways: (1) Recordings were obtained from regions that appeared normal on structural MRI scans and that were distant from the seizure focus. (2) The patients performed normally on tasks presumed to rely on the integrity of the prefrontal cortex. (3) We waited at least several hours with our recordings after the patient had any seizure.

A second limitation is the sparse sampling of different recording locations. Unlike recordings in nonhuman primates, it is impossible to move the implanted electrode to search for units. This makes our Point 3 above, the absence of topography, quite preliminary at this stage. Our main conclusions concern the nature of responses at the single-unit level recorded in the ventromedial prefrontal cortex; the larger-scale organization of such responses may be an issue better addressed with complementary techniques, such as fMRI or magnetoencephalography.
Our findings are consistent with the known connectivity of the ventral and medial prefrontal cortex. In the case of vision, the extensive connections between temporal visual regions and the prefrontal cortex (Seltzer \& Pandya, 1989) provide for an effective source of perceptual input to the orbitofrontal cortex (Fuster, Bauer, \& Jervey, 1985), wherein one finds responses to a large range of visual stimuli in both monkeys (Hikosaka \& Watanabe, 2000) and humans (Taylor, Liberzon, \& Koeppe, 2000). Whereas the prefrontal cortex participates in a widespread connectional network that allows the synthesis of emotional, mnemonic, attentional, and other cognitive functions (Barbas, 2000), the ventral and medial sectors of this region have the most extensive connections with other brain structures involved in regulating emotional behaviors and autonomic response (Öngür \& Price, 2000). The medial orbitofrontal cortex is bidirectionally connected with the amygdala (Amaral, Price, Pitkanen, \& Carmichael, 1992), ventral striatum, and basal forebrain (Ghashghaei \& Barbas, 2001), structures known to participate in the modulation of memory, attention, and other cognitive functions on the basis of the emotional significance of the stimuli being processed. Moreover, close functional relationships have been demonstrated among several of these structures in human (Morris \& Dolan, 2001) and nonhuman primates (Baxter, Parker, Lindner, Izquierdo, \& Murray, 2000; Schoenbaum, Chiba, \& Gallagher, 2000; Schultz, Tremblay, \& Hollerman, 2000; Everitt et al., 1999; Gaffan, Murray, \& Fabre-Thorpe, 1993).

Furthermore, several specifics of our findings are similar to observations of neuronal activity in the monkey prefrontal cortex: The firing rates of the neurons were quite low, and only about $15 \%$ showed responses to emotional stimuli (Rolls, 1999). Neurons in this region respond to the meaning or value of stimuli rather than to their sensory features (Hikosaka \& Watanabe 2000; Thorpe et al., 1983). Based on the firing rates of individual neurons, it was possible to predict with a mean probability of error of .34 whether the preferred stimulus category was presented or not. This level of predictability is notably lower than what has been reported for category-specific neurons in human medial temporal lobe; presumably, higher accuracy in coding is ultimately achieved by integrating across the activities of multiple neurons (Kreiman, Koch, \& Fried, 2000a, 2000b). It is possible that the weaker responses in our study resulted, at least in part, from the heterogeneity of the stimuli we used. Given the differences in complexity and meaning of each of the stimuli within an emotion category, one would expect this to result in considerable blurring of evoked responses.

Our approach here was to consider "emotion category" as a property of the stimuli rather than of the judgments that subjects make about them. Thus, for example, we would put into the "aversive" category all the stimuli to which normal subjects agree they are 
aversive. This approach analyzes the responses in the prefrontal cortex that we recorded as essentially stimulusevoked and sensory in nature. We checked that the responses we found could not be attributed to simple, confounding stimulus properties, such as luminance or color composition. However, some combination of features must of course ultimately signal the emotion categories to which the stimuli belonged. Given our choice of a very heterogeneous set of stimuli (e.g., violence, guns, illness, mutilations, etc. for the aversive category), we consider it likely that the associations between specific complex feature sets and emotion category would have been idiosyncratic and in large part acquired rather than innate, but this is a difficult issue that would require substantial future studies.

Compared to other neuroscience approaches for the study of cognition and behavior in humans, single-unit recordings provide unparalleled spatial and temporal resolution, but can be obtained only in rare neurosurgical settings (see Ojemann, Ojemann, \& Fried, 1998). Most of such investigations to date have recorded from the medial temporal lobe (Fried, Cameron, Yashar, Fong, \& Morrow, 2002; Fried, MacDonald, \& Wilson, 1997; Heit, Smith, \& Halgren, 1988), and at multiunit level (Clarke, Kalgren, Scarabin, \& Chauvel, 1995). Some of these studies found that medial temporal lobe neurons responded differentially to the expression or identity of faces or to their conjunction (Fried, Cameron, et al., 2002; Fried, MacDonald, \& Wilson, 1997). A thorough series of studies using field-potential recordings demonstrated a complex topography of responses to various biological and socially relevant stimuli in the lateral and ventral temporal cortex (Allison, Puce, \& McCarthy, 2000; Allison, Puce, Spencer, \& McCarthy, 1999). Notably, regions of the extrastriate temporal cortex were found that responded relatively selectively to the sight of human faces (Allison, Puce, Spencer, et al., 1999; McCarthy, Puce, Belger, Allison, 1999; Allison, Ginter, et al., 1994; Allison, MacCarthy, Nobre, Puce, \& Belger, 1994), paralleling similar results obtained at the single-unit level in monkeys as well as findings using functional imaging in humans. These studies are also consistent with the finding that electrical stimulation of the human posterior temporal and anterior inferior parietal cortex can interfere with labeling of facial expressions (Fried, Mateer, Ojemann, Wohns, \& Pedio, 1982). It is likely that higher-order cortices in the temporal lobe first encode the visual properties of the stimuli we used, and that this information is then subsequently passed to neurons within the ventromedial prefrontal cortex that associate the visual percept with its emotional meaning.

The precise mechanisms reflected in the neural activities we recorded remain to be fully elucidated. In light of the considerable body of findings from other studies in both humans and nonhuman primates, one proposal is that the responses we found are components of a form of associative emotional memory. It is plausible that neurons within the orbitofrontal cortex, together with those in structures like the amygdala and the ventral striatum, participate in associating purely visual perceptual representations (and perhaps perceptual representations in other sensory modalities as well) with two consequences: an emotional response, and knowledge about the emotion. Neurons within the OFC could trigger autonomic, endocrine, and other components of emotional response in virtue of their connections with hypothalamic and brainstem nuclei, and they could trigger the retrieval of knowledge about the emotion (i.e., participate in recognition of the emotion signaled by the stimulus) in virtue of their vast connections with other neocortical regions. Future studies, possibly with patients like those in the present study, could help explore this framework further by direct electrical stimulation of the same regions from which we recorded.

\section{METHODS}

\section{Subjects}

We recorded single-unit responses to visually presented stimuli in four patients who had chronically implanted depth electrodes for monitoring epilepsy. In all four patients, seizures could not be sufficiently well localized with scalp electrodes and were therefore subsequently localized with depth electrodes, from which we obtained our research recordings.

Patient 57 was a 48-year-old man with a college education. A subset of the data from this patient was presented, with a different analysis, in a prior brief communication (Kawasaki et al., 2001). Patient 66 was a 30-year-old woman with a college education whose seizures were eventually localized to the left medial temporal lobe. Patient 70 was a 39-year-old man with a high school education whose seizures were localized to bilateral posterior temporal lobes. Patient 104 was an 18-year-old man with a high school education whose seizures were localized to the right lateral dorsal prefrontal cortex.

All four patients had IQs in the normal range, normal language and memory, normal basic visual perception, and normal performances on tasks used as standards to assess frontal lobe function (Table 1), as indexed by extensive neuropsychological evaluation before neurosurgical resection (Tranel, 1996). All neuropsychological data were obtained at the time that the recordings were made. All four patients were taking antibiotic medication at the time of the recordings, but their analgesic and antiepileptic medication had been tapered (Table 1).

All subjects gave informed written consent for the implantation of the electrodes for monitoring epilepsy and, under a separate protocol, gave informed written consent to participate in research studies once the electrodes had been implanted. The research protocol 
Table 1. Subject Information

\begin{tabular}{|c|c|c|c|c|c|c|c|c|c|c|c|c|}
\hline ID & Age & Sex & Diagnosis & $H D$ & $V I Q$ & $P I Q$ & $\begin{array}{c}\text { Visual } \\
\text { Perception }\end{array}$ & $B D I$ & Language & COWA & Trailmaking & $\begin{array}{l}\text { Antiseizure Drug } \\
\text { and Dose (mg) }\end{array}$ \\
\hline 57 & 48 & M & $\begin{array}{l}\text { Simple partial seizure } \\
\text { (unknown origin) }\end{array}$ & $\mathrm{L}$ & 103 & 92 & Normal & None & Normal & Normal & Normal & $\begin{array}{l}\text { DPH } 400, \text { TGB } 48 \text {, } \\
\text { LMT } 500\end{array}$ \\
\hline 66 & 30 & $\mathrm{~F}$ & $\begin{array}{l}\text { Complex partial seizure } \\
\text { (temporal lobe epilepsy) }\end{array}$ & $\mathrm{L}$ & 125 & 110 & Normal & None & Normal & Normal & Normal & LMT 300 \\
\hline 70 & 39 & M & Complex partial seizure & $\mathrm{R}$ & 90 & 90 & Normal & None & Normal & Normal & Normal & None \\
\hline 104 & 19 & M & Complex partial seizure & $\mathrm{L}$ & 95 & 87 & Normal & Mild & Normal & Normal & Normal & None \\
\hline
\end{tabular}

ID = patient's identifier; HD = hemispheric dominance for language was verified by intracarotid amytal injection (Wada test) in all subjects. VIQ, PIQ = verbal and performance IQ from the Wechsler Adult Intelligence Scale III. Visual perception was determined by the Benton Facial Discrimination task, Judgment of Line Orientation, and the Rey-Osterrieth Complex Figure copy. Depression was assessed using the Beck Depression Inventory (BDI). Language function was assessed using a standardized battery including naming and repetition tasks. Executive function was assessed using Controlled Oral Word Association test (COWA) and the Trailmaking Test. Antiseizure drug column shows daily dosage of antiseizure medication on the day when experiment was conducted. DPH $=$ phenytoin; TGB $=$ tiagabine $\mathrm{HCl}$; LMT $=$ lamotrigine .

was approved by the federally certified Institutional Review Board of the University of Iowa.

\section{Neuroanatomical Analysis}

MR scans of the patients' brains were obtained before and after electrode implantation, and the locations of the recording sites were mapped from the postimplantation scans onto the preimplantation scans to obtain a detailed map of recording sites. Figure 1A provides an example of the images that were constructed for one of the patients to localize recording sites; similar images were constructed for the other three patients (a summary figure showing recording locations is provided in Figure 2).

\section{Stimulus Presentation}

Stimuli were 150 digital visual images drawn from a series of stimuli with known emotional valence and arousal that have been used widely in research on emotion (Lang et al., 1988) (for one of the patients, a larger stimulus set of 256 was used; see below). All images were categorized into three groups according to a combination of their mean normative ratings on valence and on arousal, namely, pleasant (valence $>6.5$ and $3<$ arousal $<6$; smiling babies, baby animals, food), aversive (valence $<3.5$ and arousal $>6$; mutilated humans or animals, scenes of war and death, snakes, spiders), and neutral ( $4<$ valence $<6$ and arousal $<6$; landscapes, pictures of household objects). We obtained the ratings in a separate session some time after the recordings had been done in order not to fatigue the subjects. Ratings of valence and arousal obtained from three subjects $(57,70$, and 104) confirmed that they rated the stimuli normally (within $2 S D$ of mean normative ratings). Subject 66 was not available for rating because we lost contact with the subject.
We attempted to choose as heterogeneous a set of stimuli within an emotion category as possible. The stimulus categories were not different in terms of their low-level properties (luminance and color composition). Although there was no difference in redness between the categories, $46 \%$ of aversive pictures that we used in this study included images of blood, and none of the other emotion categories included images of blood. Images of human faces were included in 50\% of aversive pictures, $52 \%$ of pleasant pictures, and $31 \%$ of neutral pictures.

Stimuli were presented in randomized order, with a 4-sec duration for Patient 57 and a 1-sec duration for Patients 66, 70, and 104. We used an intertrial interval that varied randomly between 5 and $8 \mathrm{sec}$ showing a single, constant, neutral complex visual image. The variable intertrial interval served to minimize any possible anticipatory responses to the next stimulus, and the complex image shown during the intertrial interval minimized luminance transients at the onset of the stimulus and served to keep the subject's cognitive state more homogeneous (e.g., to keep attention more constant). All stimuli were shown on a 14-in. LCD computer screen located approximately $1 \mathrm{~m}$ in front of the subject. For Patient 104, in order to present as many stimuli as possible within a short time, the intertrial interval was shortened to vary randomly between 800 and $1200 \mathrm{msec}$. Image presentation was controlled using either the program PsyScope (Cohen, MacWhinney, Flatt, \& Provost, 1993) running under the Macintosh Operating System or Presentation Software (Neurobehavioral Systems, Inc., Albany, CA) running under Windows 2000.

Patients were awake, alert, and reclined comfortably in a hospital bed in a dimly lit quiet room. They viewed the stimuli under free-viewing conditions. One of the experimenters, standing behind the monitor on which the stimuli were presented in the darkened room (hence not easily visible to the subject) continuously watched the subject's eyes and noted any apparent deviation in fixation or any sleepiness throughout the 
experiment. Patients were instructed to pay attention to the images on the screen and not to move or talk during the presentation. Each recording session was composed of the presentation of 60 to 256 stimuli.

\section{Recording}

Hybrid clinical research electrodes (Radionics, Inc., Burlington, MA) had two recording sites at the tip, each consisting of a cluster of four high-impedance contacts separated by $5 \mathrm{~mm}$ and a standard low-impedance contact used solely for clinical monitoring (Howard et al., 1996) (Figure 1B). The high-impedance contact locations are indicated in Figure 1A; the larger signal artifact ("ball"-shaped appearance of electrode in the top left postimplantation image) indicates the location of the low-impedance contact. The electrode design allowed recording of single-unit activity from the highimpedance contacts without introducing any additional risks to the patients.

Recorded data were band-pass filtered $(300 \mathrm{~Hz}-3 \mathrm{kHz})$ and signals whose peak amplitude exceeded a fixed threshold (approximately three times the absolute value of the noise level) were digitized at $20 \mathrm{kHz}$, sampled from -0.7 to $+1.0 \mathrm{msec}$ surrounding the first threshold crossing of the spike, and stored onto a computer hard drive. The waveforms of individual neurons were subsequently isolated using the Experimenter's Workbench and the Autocut spike sorting software (DataWave Technologies, Berthoud, CO).

\section{Statistical Analysis}

Our statistical analysis consisted of a hierarchy of two steps: (1) a numerical calculation of deviations in poststimulus firing rate from the permutation distribution of the prestimulus firing rate for each neuron, obtained from resampling epochs of its prestimulus activity (Sprent, 1998) (this first step defined a time window to which the subsequent step was restricted) and (2) calculation of selectivity for a specific emotion category.

In Step 1, we estimated the spike density function (sdf) for each category of stimuli by convolving the spike trains with a Gaussian window (200 msec SD) and then averaging over repetitions. The distribution and significance level $(p<.05)$ of the prestimulus sdf was estimated by resampling from the prestimulus activity of all trials 10,000 as many spike trains as the number of stimuli within each emotion category. We counted as significant responses only those with valid latencies, as follows. The latency was defined as the first occurrence of 10 consecutive bins of the sdf that each yielded $p<.05$. The end of the response was defined as the first time point (postlatency) at which 10 consecutive bins of the sdf yielded a nonsignificant value. It should be noted that for neurons with low spontaneous activity, the permutation distribution becomes skewed toward zero (because negative firing rates are impossible), and we consequently lose statistical power in detecting significant decreases in firing rate. Thus, our findings may be biased toward finding significant rate increases rather than decreases, especially given the generally low spontaneous firing rates we observed (mean $\pm S D: 2.3 \pm 1.9$ spikes/sec, median 1.6 spikes/ $\mathrm{sec})$. We limited ourselves to an analysis of responses that started within $1 \mathrm{sec}$ of stimulus onset. It should also be noted that our criterion for determining responsive neurons was rather conservative because it could have omitted neurons that showed increases to certain stimuli but decreases to others, thus resulting in a mean firing rate across all stimuli that failed to be significantly increased above baseline; the sparse response characteristics of the neurons we found dictated this conservative approach.

For those time epochs in which we detected significant poststimulus responses as described above, we tested response selectivity. Neurons were classified as emotion selective if the response to one emotion category, which was detected in the previous step, was significantly different $(p<.017)$ from the responses to the other two emotion categories. Distributions of spikes to each emotion category were estimated by resampling the poststimulus responses from the pool of all responses to the stimuli of that emotion category. Neurons showing responses that were significant with the first step but not selective with the second step were classified as nonselective visually responsive neurons.

For all selective neurons, to quantify the classification performance of the neurons, we used the ROC analysis. For each selective neuron, we computed the distribution of firing rates for the preferred stimuli and for the nonpreferred stimuli. From the distribution of firing rates, the probability of correct detection $\left(P_{\mathrm{CD}}\right)$ and the probability of false alarm $\left(P_{\mathrm{FA}}\right)$ were evaluated by sliding the threshold $(T)$ over the whole range of spike rates. The probability of misclassification $\left(P_{\text {error }}\right)$ was computed as follows:

$$
P_{\text {error }}(T)=1 / 2 P_{\mathrm{FA}}(T)+1 / 2\left(1-P_{\mathrm{CD}}(T)\right)
$$

The lowest possible probability of error $\left(P_{\text {error }}\right)$ that the ideal observer could achieve is defined as the minimum value of this function. A value of $P_{\text {error }}$ at .5 corresponds to chance performance, whereas a value of $P_{\text {error }}=0$ corresponds to perfect discrimination (i.e., it is possible to predict exactly on the basis of the spikes recorded whether the stimulus belonged to the preferred emotion category or not).

In one subject (Subject 104) we investigated whether neural responses might be better explained by simpler 
dimensional properties of the stimuli rather than their categorization into basic emotions. This subject viewed a total of 256 pictures of various emotional contents including the standard 150 pictures used for analysis by emotion category (which all other subjects saw). The distribution of valence and arousal of all stimuli are plotted in Figure 5A. For each neuron that yielded significant responses according to the two-step statistical analysis by emotion category described above, we also calculated Spearman rank-order correlations between spike count (within the time window of the significant response) and valence or arousal scores of the stimuli. Correlation coefficients and associated probabilities were calculated by grouping the stimuli into sets on the basis of valence or arousal. Correlations with valence were computed on the moderate arousal group ("ma," $4.5<$ arousal $<5.5, n=106$ ) (Figure 5A, horizontal rectangle). Similarly, correlations with arousal were computed on the moderate valence group ("mv," $4.5<$ valence $<5.5, n=83$ ) (Figure 5A, vertical rectangle) to minimize possible interactions between valence and arousal.

\section{Acknowledgments}

We thank Kodi Scheer, Yota Kimura, John Brugge, Igor Volkov, Quingyu Li, and Soman Puzhankara for help with the experiments and data analysis; Mark Granner for providing epilepsy center services; and Daniel Tranel for help with background neuropsychological testing. We thank our epilepsy surgery patients for their generosity in participating in this research. This study was supported by grants from the EJLB Foundation, the Klingenstein Fund, and the James S. McDonnell Foundation.

Reprint requests should be sent to Ralph Adolphs, HSS 22877, Caltech, Pasadena, CA 91125, or via e-mail: radolphs@ hss.caltech.edu.

\section{REFERENCES}

Allison, T., Ginter, H., McCarthy, G., Nobre, A. C., Puce, A., Luby, M., \& Spencer, D. D. (1994). Face recognition in human extrastriate cortex. Journal of Neurophysiology, 71, 821-825.

Allison, T., McCarthy, G., Nobre, A., Puce, A., \& Belger, A. (1994). Human extrastriate visual cortex and the perception of faces, words, numbers, and colors. Cerebral Cortex, 4 , 544-554.

Allison, T., Puce, A., \& McCarthy, G. (2000). Social perception from visual cues: Role of the STS region. Trends in Cognitive Sciences, 4, 267-278.

Allison, T., Puce, A., Spencer, D., \& McCarthy, G. (1999). Electrophysiological studies of human face perception. I. Potentials generated in occipitotemporal cortex by face and non-face stimuli. Cerebral Cortex, 9, 415-430.

Amaral, D. G., Price, J. L., Pitkanen, A., \& Carmichael, S. T. (1992). Anatomical organization of the primate amygdaloid complex. In J. P. Aggleton (Ed.), The amygdala: Neurobiological aspects of emotion, memory, and mental dysfunction (pp. 1-66). New York: Wiley-Liss.
Barbas, H. (2000). Connections underlying the synthesis of cognition, memory, and emotion in primate prefrontal cortices. Brain Research Bulletin, 52, 319-330.

Baxter, M. G., Parker, A., Lindner, C. C. C., Izquierdo, A. D., \& Murray, E. A. (2000). Control of response selection by reinforcer value requires interaction of amygdala and orbital prefrontal cortex. Journal of Neuroscience, 20, 4311-4319.

Bechara, A., Damasio, A. R., Damasio, H., \& Anderson, S. W. (1994). Insensitivity to future consequences following damage to human prefrontal cortex. Cognition, 50, 7-15.

Blair, R. J. R., Morris, J. S., Frith, C. D., Perrett, D. I., \& Dolan, R. J. (1999). Dissociable neural responses to facial expressions of sadness and anger. Brain, 122, 883-893.

Borod, J. C., Cicero, B. A., Obler, L. K., Welkowitz, J., Erhan, H. M., Santschi, C., Grunwald, I. S., Agosti, R. M., \& Whalen, J. R. (1998). Right hemisphere emotional perception-Evidence across multiple channels. Neuropsychology, 12, 446-458.

Canli, T., Zhao, Z., Desmond, J. E., Glover, G., \& Gabriel, J. D. E. (1999). fMRI identifies a network of structures correlated with retention of positive and negative emotional memory. Psychobiology, 27, 441-452.

Cavada, C., \& Schultz, W. (2000). The mysterious orbitofrontal cortex. Cerebral Cortex, 10, 205-342.

Clarke, J. M., Halgren, E., Scarabin, J. M., \& Chauvel, P. (1995). Auditory and visual representations in human prefrontal cortex as revealed by stimulus-evoked spike-wave complexes. Brain, 118, 473-484.

Cohen, J. D., MacWhinney, B., Flatt, M., \& Provost, J. (1993). PsyScope: A new graphic interactive environment for designing psychology experiments. Behavior Research Methods, Instruments, \& Computers, 25, 257-271.

Damasio, A. R. (1994). Descartes' error: Emotion, reason, and the human brain. New York: Grosset/Putnam.

Damasio, A. R., Grabowski, T. J., Bechara, A., Damasio, H., Ponto, L. L. B., Parvizi, J., \& Hichwa, R. D. (2000). Subcortical and cortical brain activity during the feeling of self-generated emotions. Nature Neuroscience, 3, 1049-1056.

Davidson, R. J. (1999). Anterior cerebral asymmetry and the nature of emotion. Brain and Cognition, 20, 125-151.

Davidson, R. J., \& Irwin, W. (1999). The functional neuroanatomy of emotion and affective style. Trends in Cognitive Sciences, 3, 11-21.

Drevets, W. C., Price, J. L., Simpson, J. R., Todd, R. D., Reich, T., Vannier, M., \& Raichle, M. E. (1997). Subgenual prefrontal cortex abnormalities in mood disorders. Nature, 386, 824-827.

Everitt, B. J., Parkinson, J. A., Olmstead, M. C., Arroyo, M., Robledo, P., \& Robbins, T. W. (1999). Associative processes in addiction and reward. The role of amygdala-ventral striatal subsystems. Annals of the New York Academy of Sciences, 877, 412-438.

Fried, I., Cameron, K. A., Yashar, S., Fong, R., \& Morrow, J. W. (2002). Inhibitory and excitatory responses of single neurons in the human medial temporal lobe during recognition of faces and objects. Cerebral Cortex, 12, 575-584.

Fried, I., MacDonald, K. A., \& Wilson, C. L. (1997). Single neuron activity in human hippocampus and amygdala during recognition of faces and objects. Neuron, 18, $753-765$. 
Fried, I., Mateer, C., Ojemann, G., Wohns, R., \& Fedio, P. (1982). Organization of visuospatial functions in human cortex; evidence from electrical stimulation. Brain, 105, 349-371.

Fuster, J. M., Bauer, R. H., \& Jervey, J. P. (1985). Functional interactions between inferotemporal and prefrontal cortex in a cognitive task. Brain Research, 330, 299-307.

Gaffan, D., Murray, E. A., \& Fabre-Thorpe, M. (1993). Interaction of the amygdala with the frontal lobe in reward memory. European Journal of Neuroscience, 5, 968-975.

Ghashghaei, H. T., \& Barbas, H. (2001). Neural interaction between the basal forebrain and functionally distinct prefrontal cortices in the rhesus monkey. Neuroscience, 103, 593-614.

Harmer, C. J., Thilo, K. V., Rothwell, J. C., \& Goodwin, G. M. (2001). Transcranial magnetic stimulation of medial-frontal cortex impairs the processing of angry facial expressions. Nature Neuroscience, 4, 17-18.

Heit, G., Smith, M. E., \& Halgren, E. (1988). Neural encoding of individual words and faces by the human hippocampus and amygdala. Nature, 333, 773-775.

Hikosaka, K., \& Watanabe, M. (2000). Delay activity of orbital and lateral prefrontal neurons of the monkey varying with different rewards. Cerebral Cortex, 10, 263-271.

Hornak, J., Rolls, E. T., \& Wade, D. (1996). Face and voice expression identification in patients with emotional and behavioral changes following ventral frontal lobe damage. Neuropsychologia, 34, 247-261.

Howard, M. A., Volkov, I. O., Granner, M. A., Damasio, H. M., Ollendieck, M. C., \& Bakken, H. E. (1996). A hybrid clinical-research depth electrode for acute and chronic in-vivo microelectrode recording of human brain neurons. Journal of Neurosurgery, 84, 129-132.

Kawasaki, H., Adolphs, R., Kaufman, O., Damasio, H., Damasio, A. R., Granner, M., Bakken, H., Hori, T., \& Howard, M. A. (2001). Single-unit responses to emotional visual stimuli recorded in human ventral prefrontal cortex. Nature Neuroscience, 4, 15-16.

Kreiman, G., Koch, C., \& Fried, I. (2000a). Category-specific visual responses of single neurons in the human medial temporal lobe. Nature Neuroscience, 3, 946-953.

Kreiman, G., \& Koch, C., Fried, I. (2000b). Imagery neurons in the human brain. Nature, 408, 357-361.

Lang, P. J., Oehman, A., \& Vaitl, D. (1988). The international affective picture system. Gainesville: University of Florida Press.

Marinkovic, K., Trebon, P., Chauvel, P., \& Halgren, E. (2000). Localised face processing by the human prefrontal cortex: Face-selective intracerebral potentials and post-lesion deficits. Cognitive Neuropsychology, 17, 187-199.

McCarthy, G., Puce, A., Belger, A., \& Allison, T. (1999). Electrophysiological studies of human face perception II: Response properties of face-specific potentials generated in occipitotemporal cortex. Cerebral Cortex, 9, 431-444.

Morris, J. S., \& Dolan, R. J. (2001). Involvement of human amygdala and orbitofrontal cortex in hunger-enhanced memory for food stimuli. Journal of Neuroscience, 21, 5304-5310.

Northoff, G., Richter, A., Gessner, M., Schlagenhauf, F., Fell, J., Baumgart, F., Kaulisch, T., Koetter, R., Stephan, K. E., Leschinger, A., Hagner, T., Bargel, B.,
Witzel, T., Hinrichs, H., Bogerts, B., Scheich, H., \& Heinze, H.-J. (2000). Functional dissociation between medial and lateral prefrontal cortical spatiotemporal activation in negative and positive emotions: A combined fMRI/MEG study. Cerebral Cortex, 10, 93-107.

O'Doherty, J., Kringelbach, M. L., Rolls, E. T., Hornak, J., \& Andrews, C. (2001). Abstract reward and punishment representations in the human orbitofrontal cortex. Nature Neuroscience, 4, 95-102.

Ojemann, G. A., Ojemann, S. G., \& Fried, I. (1998). Lessons from the human brain: Neuronal activity related to cognition. Neuroscientist, 4, 285-300.

Öngür, D., \& Price, J. L. (2000). The organization of networks within the orbital and medial prefrontal cortex of rats, monkeys, and humans. Cerebral Cortex, 10, 206-219.

Roesch, M. R., \& Olson, C. R. (2004). Neuronal activity related to reward value and motivation in primate frontal cortex. Science, 304, 307-310.

Rolls, E. T. (1999). The brain and emotion. Oxford: Oxford University Press.

Rolls, E. T., Critchley, H. D., Mason, R., \& Wakeman, E. A. (1996). Orbitofrontal cortex neurons: Role in olfactory and visual association learning. Journal of Neurophysiology, 75, 1970-1981.

Royet, J. P., Zald, D., Versace, M., Costes, N., Lavenne, F., Koenig, O., \& Gervais, R. (2000). Emotional responses to pleasant and unpleasant olfactory, visual, and auditory stimuli: A positron emission tomography study. Journal of Neuroscience, 20, 7752-7759.

Schoenbaum, G., Chiba, A. A., \& Gallagher, M. (2000). Changes in functional connectivity in orbitofrontal cortex and basolateral amygdala during learning and reversal training. Journal of Neuroscience, 20, 5179-5189.

Schultz, W., Tremblay, L., \& Hollerman, J. R. (2000). Reward processing in primate orbitofrontal cortex and basal ganglia. Cerebral Cortex, 10, 272-283.

Seltzer, B., \& Pandya, D. N. (1989). Frontal lobe connections of the superior temporal sulcus in the rhesus monkey. Journal of Comparative Neurology, 281, 97-113.

Sprent, P. (1998). Data-driven statistical methods. New York: Chapman \& Hall.

Taylor, S. F., Liberzon, I., \& Koeppe, R. A. (2000). The effect of graded aversive stimuli on limbic and visual activation. Neuropsychologia, 38, 1415-1425.

Thorpe, S. J., Rolls, E. T., \& Maddison, S. (1983). The orbitofrontal cortex: Neuronal activity in the behaving monkey. Experimental Brain Research, 49, 93-115.

Tranel, D. (1996). The Iowa-Benton school of neuropsychological assessment. In I. Grant \& K. M. Adams (Eds.), Neuropsychological assessment of neuropsychiatric disorders (2nd ed.). New York: Oxford University Press, 1996, p. 81-101.

Vuilleumier, P., Armony, J. L., Driver, J., \& Dolan, R. J. (2001). Effects of attention and emotion on face processing in the human brain. An event-related fMRI study. Neuron, 30, 829-841.

Wallis, J., \& Miller, E. K. (2003). Neuronal activity in primate dorsolateral and orbital prefrontal cortex during performance of a reward preference task. European Journal of Neuroscience, 18, 2069-2081. 